\title{
Pathological diagnosis of cirrhotic ascites in a Caucasian Dog
}

\author{
Samson Eneojo Abalakaa; Sylvester Sunday Obetab; Uchenna Stephen Asogwac; Nuhu \\ Abdulazeez Sania; Idoko Sunday Idoko; Sunday Augustine Ejeh ${ }^{d}$; Ahmadu Salehe; Oremeyi \\ Zainab Tenuche \\ a Department of Veterinary Pathology, University of Abuja, Abuja, Nigeria \\ b Department of Veterinary Parasitology and Entomology, University of Abuja, Abuja, Nigeria \\ c Faculty of Veterinary Medicine, University of Nigeria, Nsukka, Nigeria \\ d Department of Veterinary Physiology and Biochemistry, University of Abuja, Abuja, Nigeria \\ e Department of Veterinary Pathology, Ahmadu Bello University, Zaria, Nigeria
}


Available online at www.refaad.com

VMPH 2(2); 2021: 31-36

Research Article

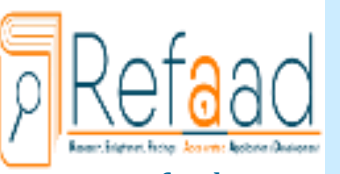

www.refaad.com
Veterinary Medicine and Public Health Journal (VMPH)

Journal Homepage: https://www.refaad.com/views/vmph/home.aspx

\title{
Pathological diagnosis of cirrhotic ascites in a Caucasian Dog
}

\section{Samson Eneojo Abalaka*a; Sylvester Sunday Obetab; Uchenna Stephen Asogwac; Nuhu Abdulazeez Sania; Idoko Sunday Idoko; ${ }^{a}$ Sunday Augustine Ejeh"; Ahmadu Salehe; Oremeyi Zainab Tenuche ${ }^{a}$}

\author{
a Department of Veterinary Pathology, University of Abuja, Abuja, Nigeria \\ ${ }^{\mathrm{b}}$ Department of Veterinary Parasitology and Entomology, University of Abuja, Abuja, Nigeria \\ c Faculty of Veterinary Medicine, University of Nigeria, Nsukka, Nigeria \\ ${ }^{\mathrm{d}}$ Department of Veterinary Physiology and Biochemistry, University of Abuja, Abuja, Nigeria \\ e Department of Veterinary Pathology, Ahmadu Bello University, Zaria, Nigeria
}

* Corresponding author: Abalaka, S. E. E-mail: samson.abalaka@uniabuja.edu.ng

How to cite this article: Abalaka, S. E. et al., Pathological diagnosis of cirrhotic ascites in a Caucasian Dog. Veterinary Medicine and Public Health Journal 2(2); 2021: 31-36.

DOI: https://doi.org/10.31559/vmph2021.2.2.1 Received Date: 2/2/2021 Revised Date: 3/4/2021 Accepted Date: 4/5/2021

\begin{abstract}
Although ascites is widely reported in dogs, those of cirrhotic ascites are scanty, especially in Abuja, Nigeria, hence the need for this report on cirrhotic ascites in a 2-year-old Caucasian dog. The reported complaints of anorexia, weakness, and distended abdomen before the presentation for proper veterinary attention led to a tentative diagnosis of suspected liver-induced ascites. The dog later died while receiving treatment necessitating the performance of a post-mortem examination on the severely emaciated carcass, which had pale mucous membranes, distended abdomen, and slightly oedematous distal limbs. Grossly, the lungs had multiple areas of congestion with visible hydrothorax and hydroperitoneum while the liver was shrunken, firm, nodular, misshapen, and haemorrhagic. Pulmonary congestion and oedema characterized the lung microscopically. The liver showed hepatic haemorrhage, cytoplasmic vacuolation, and necrosis with mononuclear cellular infiltration, fibrous connective tissue proliferation, and nests of apparently normal hepatocytes. The positive alpha-smooth muscle actin and vimentin immunoreactivity confirmed the hepatic fibrosis. Microscopic renal lesions indicated nephropathy. Consequently, the definitive diagnosis became cirrhotic ascites. The observed liver pathology indicated end-stage liver damage responsible for the ascites and eventual death of the dog. Prompt diagnosis with appropriate treatment is very crucial in the prognosis of cirrhotic ascites in affected dogs.
\end{abstract}

Keywords: Caucasian dog; Cirrhotic liver, Ascites; Pathology. 


\section{Introduction}

Ascites is the accumulation of serous fluid within the peritoneal cavity, which may be due to chronic hepatic failure, congestive heart failure, malnutrition, ankylostomiasis, protein-losing enteropathy, or nephritic syndrome in dogs (Regmi and Shah 2017) indicative of pre-hepatic, hepatic, or post-hepatic ascites. Ascites from liver cirrhosisinduced portal hypertension increases circulating nitric oxide beyond threshold levels resulting in vasodilatation to elevate plasma vasoconstrictor sodium-retentive hormones levels leading to renal dysfunction (Chiejina et al. 2020). Therefore, ascites denotes a serious underlying disorder requiring prompt and rationale treatment based on proper diagnosis (Nwoha 2019).

Ascites is usually diagnosed via haematological and biochemical evaluations, cytological analysis of the abdominal fluid, and radiography or ultrasonography among others (Nwoha 2019). Nevertheless, the condition presents diagnostic difficulties to average veterinarians due to the apparent lack of information on the specific cause and factors predisposing to the condition in affected dogs (Ihedioha et al. 2013). Therefore, a holistic approach to determine the aetiology of ascites often encountered in dogs should also include both antemortem and post-mortem pathological evaluations of such cases as attempted in the present case. Similarly, although ascites in dogs is well documented (Regmi and Shah 2017; Nwoha 2019), reports of cirrhotic liver-induced ascites are particularly scarce hence the need for this present case report.

\section{Case presentation}

\section{History:}

A 2-year-old Caucasian security dog, mainly on homemade food, was referred to a private veterinary clinic with chief complaints of swollen abdomen, weakness, and sores on the limbs, which became obvious about two weeks prior to the presentation. The client, who suspected ascites of unknown origin before seeking veterinary attention, initially managed the dog at home. The vaccination and deworming records of the dog was reportedly up-to-date. Clinical examination showed normal rectal temperature with dyspnoea, and tachycardia as well as palpable popliteal lymph nodes, pale mucous membrane with sunken eyes, and extensively distended abdomen with fluid wave during percussion and ballottement. Others included mild oedematous distal extremity of the four limbs with multiple sores, which all led to a tentative diagnosis of ascites of suspected hepatic origin. Treatment included Spironolactone $(2 \mathrm{mg} / \mathrm{kg}, \mathrm{PO} \mathrm{x}$ 1/52), Furosemide (2 $\mathrm{mg} / \mathrm{kg}$, PO $x \mathrm{1} / 52)$,
Oxytetracycline LA (20 mg/kg, IM x 2/7), vitamin BComplex (2 ml, IM x 5/7), and Silymarin (50 mg/kg, PO x 1/52). However, the dog died some 48 hours after the commencement of the treatment.

\section{Pathological evaluation:}

The post-mortem examination of the carcass followed standard procedures that also involved the fixing of the harvested tissues in $10 \%$ neutral buffered formalin and processing for histopathological evaluation as described by Bancroft and Cook (1994). Similarly, the alphasmooth muscle actin ( $\alpha$-SMA) and vimentin immunohistochemical evaluation of the liver was based on the Avidin-Biotin Complex (ABC) method (Hsu et al. 1981) following the obtained histopathological results.

The post-mortem evaluation revealed severely emaciated carcass with pale ocular and oral mucus membranes, markedly swollen abdomen, soiled perineum, and oedematous limbs with bruises. Others included gelatinous subcutaneous fat, atrophied sub-mandibular lymph nodes, and prominent ribs with atrophied intercostals muscles. There was amber coloured hydrothorax $(115 \mathrm{ml})$ containing fibrin that clotted few minutes after exposure to air (Fig. 1). We also observe bilateral multiple congested areas within the lungs (Fig. 1) in addition to the presence of small amount of froth within the trachea that proceeded into the bronchioles, including petechiae of the coronary fat.

Similarly, we obtained about eight litres $(8 \mathrm{~L})$ of clear straw-coloured hydroperitoneum also containing fibrin that clotted few minutes upon exposure to air (Fig. 2). The liver was grossly atrophied, firm, nodular, misshapen, and haemorrhagic; especially the left cranial lobe, with engorged gall bladder and fibrinous coverings (Fig. 3 ). The heart and kidneys showed no obvious gross lesions. However, there was moderate diffuse pulmonary congestion and haemorrhage with fibrincontaining oedema (Fig. 4) within the lungs microscopically. The liver showed marked multifocal hepatic necrosis with haemorrhages and mononuclear cellular infiltration with cytoplasmic vacuolations, as well as portal, periportal and bridging fibrosis around nests of apparently normal hepatocytes (Fig 5). There was a positive immunoreactivity of the portal, periportal, and bridging fibrosis to $\alpha$-SMA and vimentin antibodies (Figs. 6 and 7). Although we observed no obvious gross kidney lesions, there were diffuse widened Bowman's spaces and tubular necrosis with eosinophilic contents microscopically (Fig. 8). The heart presented no obvious histopathological changes (Fig. 9). 


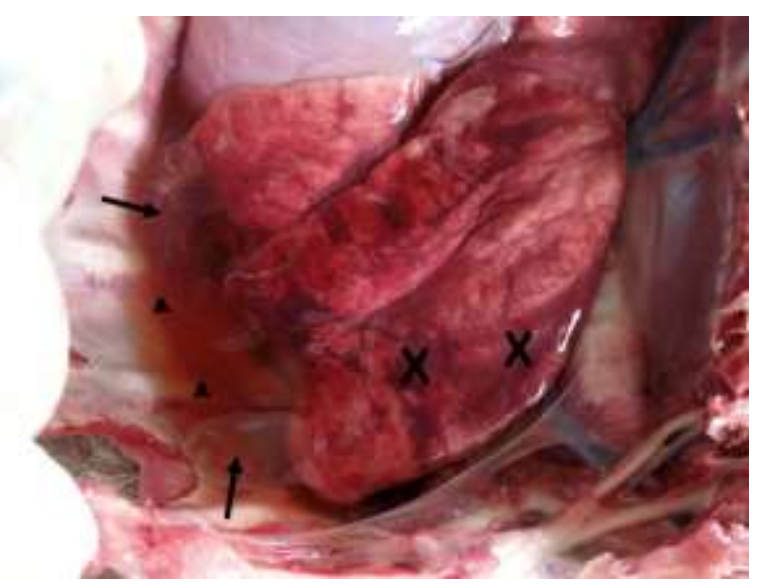

Figure (1): Photograph of the thoracic cavity of a 2-year-old Caucasian dog with cirrhotic ascites. Note the straw-coloured hydrothorax (arrowheads) with fibrin (arrows) and the lung with multiple congested areas (X).

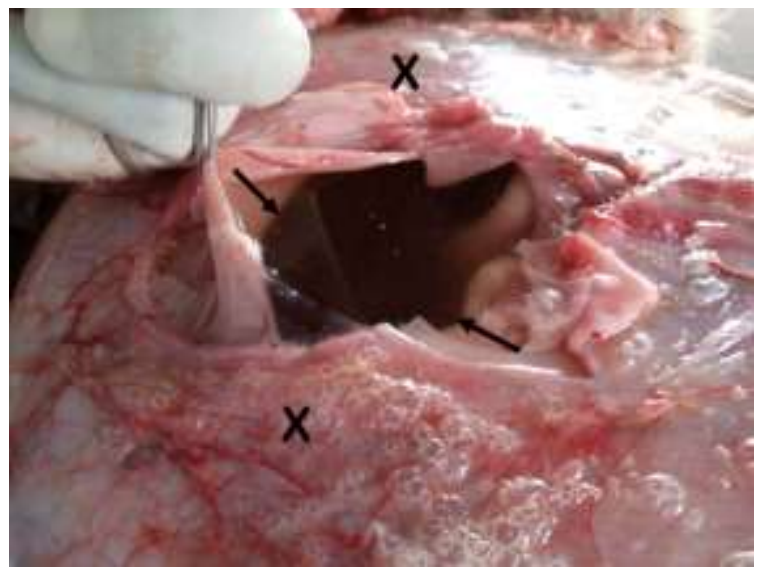

Figure (2): Photograph of the abdominal cavity of a 2-year-old Caucasian dog with cirrhotic ascites. Note the abdomen (X) containing straw-coloured fluid (arrows)

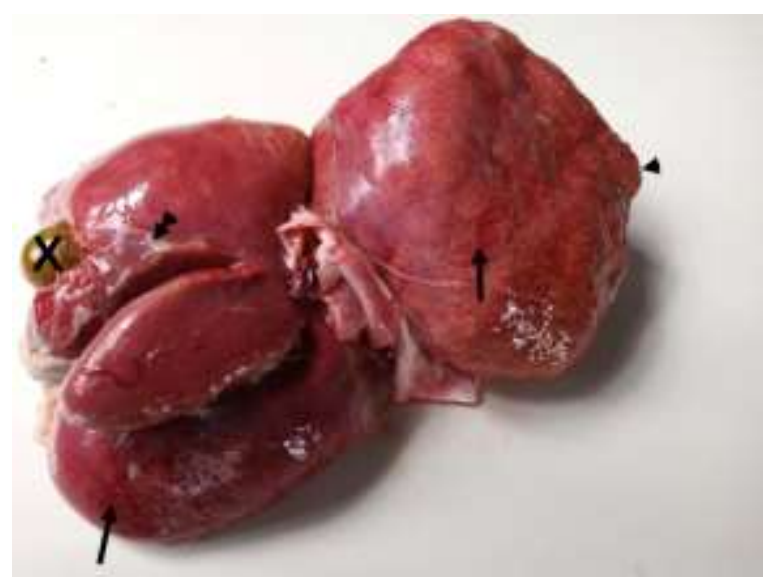

Figure (3): Photograph of the liver of a 2-year-old Caucasian dog with cirrhotic ascites. Note the shrunken, nodular, and misshapen liver (arrowhead) with hepatic haemorrhages (arrows), engorged bladder (X) and fibrinous covering (double arrowheads) 


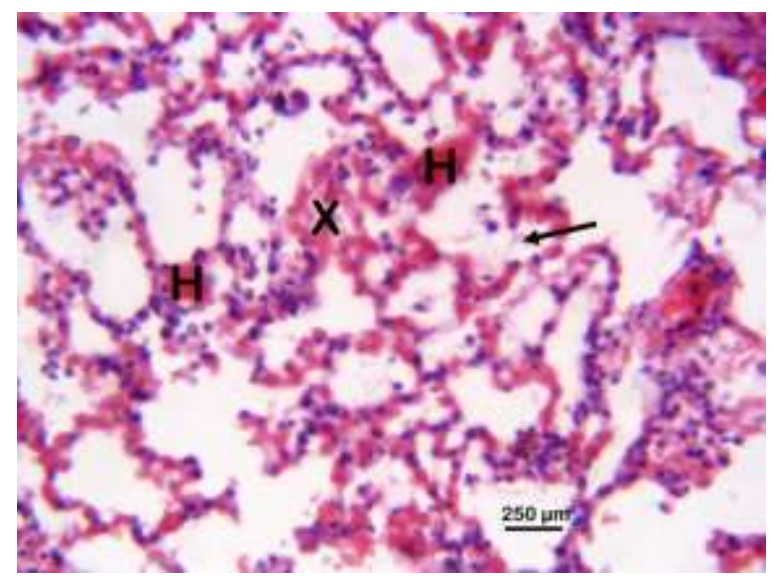

Figure (4): Photomicrograph of the lung of a 2-year-old Caucasian dog with cirrhotic ascites. Note the pulmonary congestion $(\mathrm{H})$, haemorrhages $(\mathrm{X})$, and fibrin-containing oedema (arrows). H \& E.

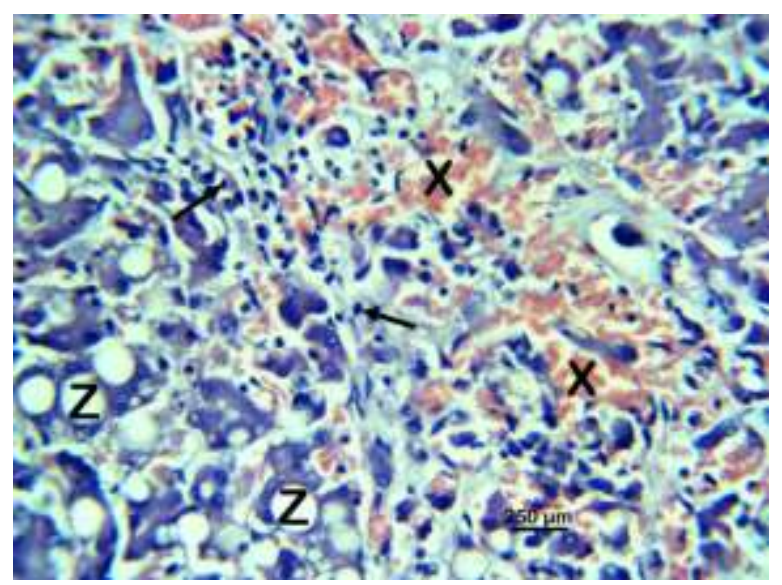

Figure (5): Photomicrograph of the liver of a 2-year-old Caucasian dog with cirrhotic ascites. Note the hepatic haemorrhages $(\mathrm{X})$, hepatic necrosis with hepatic mononuclear cellular infiltration (arrows), and hepatic cytoplasmic vacuolation (Z). H \& E.

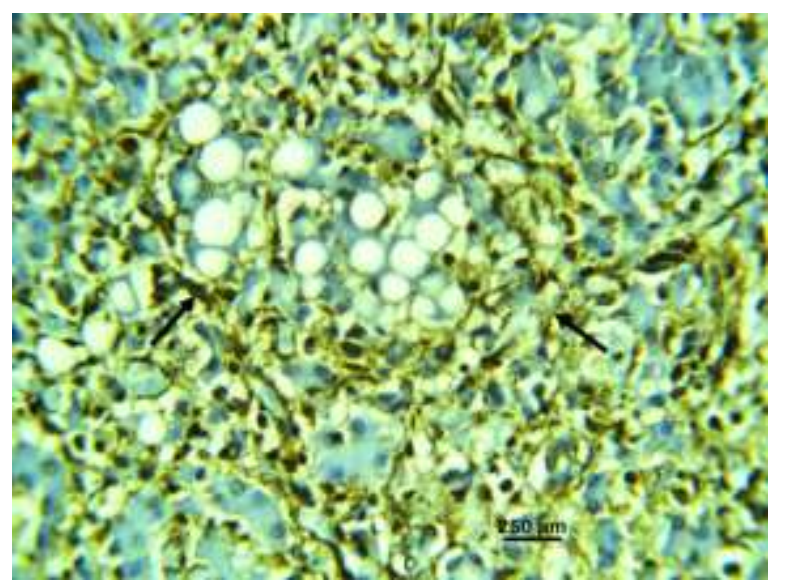

Figure (6): Photomicrograph of the liver of a 2-year-old Caucasian dog with cirrhotic ascites. Note the positive reaction of the bridging hepatic septal fibrous tissues to alpha-smooth muscle actin ( $\alpha$-SMA) immunohistochemistry (arrows). $\alpha$-SMA. 


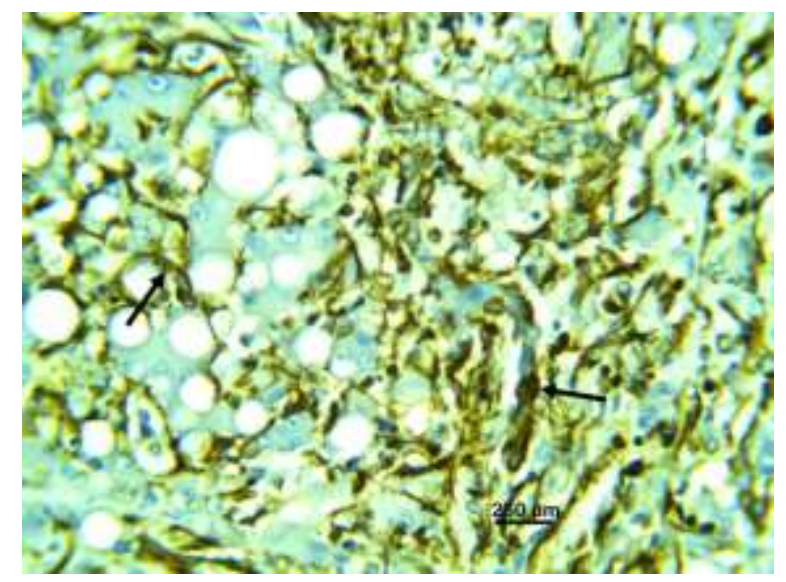

Figure (7): Photomicrograph of the liver of a 2-year-old Caucasian dog with cirrhotic ascites. Note the positive reaction of the bridging hepatic septal fibrous tissues to vimentin immunohistochemistry (arrows). Vimentin.

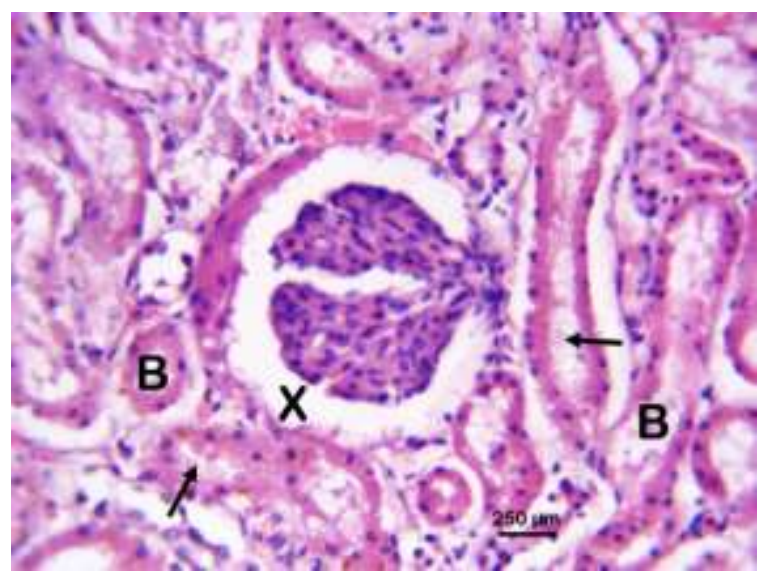

Figure (8): Photomicrograph of the kidney of a 2-year-old Caucasian dog with cirrhotic ascites. Note the diffuse widened Bowman's capsule (X) and tubular epithelial necrosis (B) with eosinophilic tubular contents (arrows). H \& E.

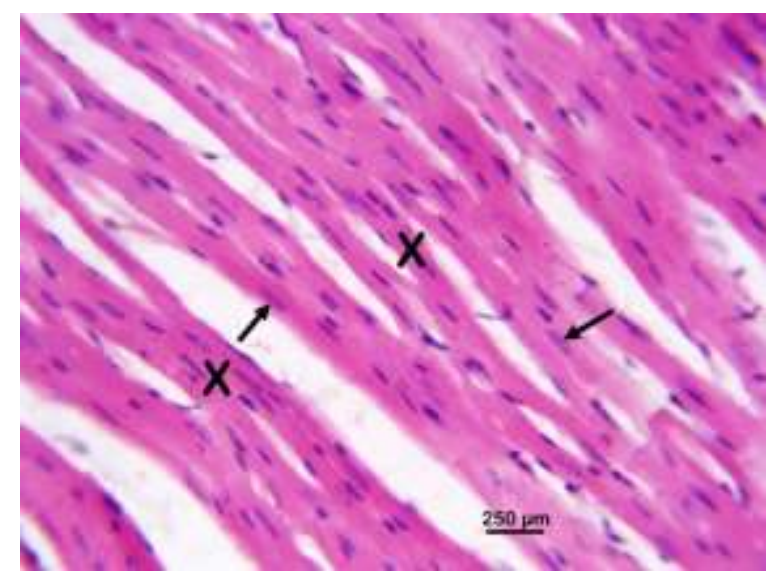

Figure (9): Photomicrograph of the heart of a 2-year-old Caucasian dog with cirrhotic ascites. Note the apparently normal myocardiocytes $(\mathrm{X})$ and intact myocardial nucleus (arrows). H \& E.

\section{Discussion}

The observed clinical signs of fluid distended abdomen with the loss of body condition suggested ascites. The instituted treatment notwithstanding, thorough laboratory-based definitive diagnosis is usually the hallmark of any appropriate treatment in affected dogs. The Spironolactone and Furosemide combination aimed to achieve greater sodium excretion while Silymarin administration was due to its acclaimed anti-inflammatory and hepatoprotective activities. The clear straw-coloured hydroperitoneum possibly indicated a long-standing ascites of hepatic origin (Nwoha 2019). The shrunken, nodular, and misshapen liver characterized histopathologically by the proliferated fibrous tissues, inflammatory cellular infiltration, nests of apparently normal hepatocytes, and distorted hepatic architecture indicated cirrhosis or end-stage liver damage. The positive hepatic $\alpha$-SMA and vimentin reactivity in the present case suggested the presence of hepatic stellate cells, 
which are of mesenchymal cellular origin (Milijana et al. 2009) and known for producing the hepatic extracellular matrix in hepatic fibrosis (SmithCortinez et al. 2021). Chronic hepatitis probably induced the observed hepatic fibrosis according to Eulenberg and Lidbury (2018). The hepatic cytoplasmic vacuolation suggested fatty change within the cirrhotic liver. The histomorphological changes in the liver of the dog might have caused portal hypertension, arterial vasodilatation, and neurohumoral activation leading to sodium and water retention and the fluid accumulation (Gentilini et al. 2002).

The absence of apparent cellular changes within the myocardium further points to liver compromise as the cause of the observed ascites. The observed widened Bowman's spaces and tubular necrosis with eosinophilic tubular content indicated nephropathy in the present case. Therefore, the dog most probably died from hypovolemic shock arising from extra-vascular fluid redistribution. The post-mortem evaluation of the present case revealed cirrhotic ascites whose diagnosis proved daunting during the ante-mortem evaluation occasioned by the non-specificity of the manifested signs and the apparent lack of thorough confirmatory laboratory investigations. Therefore, early diagnosis and appropriate treatment remains the only way to improve the prognosis of the case in affected dogs.

Acknowledgements: We sincerely acknowledge the contribution of Mr. Jonathan Madukwe of the Histopathology Unit, National Hospital, Abuja, Nigeria, to the work.

Conflict of interests: The authors declared no conflict of interests.

\section{References}

1. Bancroft, J.D. \& Cook, H.C. (1994). Manual of Histological Techniques and Their Diagnostic Application. Churchill Livingstone; London. Pp. 289305.

2. Chiejina, M., Kudaravalli P. \& Samant. H. (2020). "Ascites". StatPearls [Internet]. Available from:
https://www.ncbi.nlm.nih.gov/books/NBK470482/.

Accessed 15 April 2021.

3. Eulenberg, V.M. \& Lidbury, J.A. (2018). Hepatic Fibrosis in Dogs. J Vet Intern Med. 32(1): 26-41. doi: 10.1111/jvim.14891.

4. Gentilini, P., Vizzutti, F., Gentilini, A., Zipoli, M., Foschi, M. \& Romanelli, R.G. (2002). Update on ascites and hepatorenal syndrome. Dig Liver Dis. 34(8): 592-605. doi: 10.1016/s1590-8658(02)80094-9.

5. Hsu, S.M., Raine, L. \& Fanger, H. (1981). A comparative study of the peroxidase-antiperoxidase method and an avidin-biotin complex method for studying polypeptide hormones with radioimmunoassay antibodies. Am J Clin Pathol. 75(5): 734-738. doi: 10.1093/ajcp/75.5.734.

6. Ihedioha, J.I., Anosa, V.O. \& Esievo, K.A.N. (2013). Prevalence of and clinicopathologic findings associated with ascites in dogs in Enugu State, Nigeria. Comp Clin Pathol. 22: 185-193. https://doi.org/10.1007/s00580-011-1385-2.

7. Milijana, K., Gledić, D, Kukolj, V., Knežević, D.J., Tatjana, J.M.C. \& Sanja, A. (2009). Expression of $\alpha$ SMA, desmin and vimentin in canine liver with fibrosis. Acta Veterinaria (Beograd), 59(4): 361-370. doi: 10.2298/AVB0904361K.

8. Nwoha, R.J.O. (2019). Review on ascites in pets. In: Bekoe, S.O., Saravanan, M., Adosraku, R.K. \& Ramkumar, P.K. (eds.). Veterinary Medicine and Pharmaceuticals. IntechOpen. doi: 10.5772/intechopen.84767. Available from: https://www.intechopen.com/books/veterinary-medicineand-pharmaceuticals/review-on-ascites-in-pets. Accessed 3 January 2021.

9. Regmi, B. \& Shah, M.K. (2017). A case study on ascites of hepatic origin and their proper management in a male German shepherd dog. Int J Appl Sci Biotechnol. 5(4): 555-558. doi: 10.3126/ijasbt.v5i4.18768

10. Smith-Cortinez, N., Fagundes, R.R., Gomez, V., Kong, D., de Waart, D.R., Heegsma, J., Sydor, S., Olinga, P., de Meijer, V. E., Taylor, C.T., Bank, R., Paulusma, C.C. \& Faber, K.N. (2021). Collagen release by human hepatic stellate cells requires vitamin $\mathrm{C}$ and is efficiently blocked by hydroxylase inhibition. The FASEB Journal, $35 \quad$ (2): e21219. https://doi.org/10.1096/fj.202001564RR. 